\title{
Activismo ambiental e incidencia para la adaptación al cambio climático en Acapulco
}

\section{Environmental activism and advocacy for climate change adaptation in Acapulco}

\author{
Ramiro Salvador Gómez Villerías • \\ Erick Alfonso Galán Castro"• \\ Manuel Ignacio Ruz Vargas".•
}

\begin{abstract}
Resumen
Partiendo de experiencias de participación de actores de la sociedad civil, nos proponemos a desarrollar un diagnóstico de la relación sociedad-Estado en políticas de adaptación al cambio climático en Acapulco, Guerrero. La información fue recabada mediante entrevistas en profundidad semiestructuradas. La investigación se codificó y categorizó identificando uno o más pasajes de texto con un tema y relacionándolo con un código. Encontramos una fragmentación de agendas y mecanismos de acción pública de los actores civiles en un entorno político caracterizado por un autoritarismo subnacional que impide la construcción de agendas políticas disidentes, $y$ una falta de conocimiento especializado sobre el problema del cambio climático por parte de los activistas locales. Para que la información de la ciencia climática sea asimilada, debe comunicarse activamente con el lenguaje apropiado, buscando mecanismos participativos que generen producción de datos confiables $y$ transparentes, $y$ que fortalezcan la emergencia de una agenda ambientalista común.
\end{abstract}

Palabras clave: cambio climático, ambientalismo, activismo, política ambiental, sociedad civil.

\begin{abstract}
Based on experiences of participation by civil society actors, we propose to develop a diagnosis of the relationship between society and the State in policies of adaptation to climate change in Acapulco, Guerrero. The information was collected through semi-structured in-depth interviews. The information was coded and categorized by identifying one or more text passages with a theme and relating it to a code. We found a fragmentation of agendas and mechanisms for public action by civil actors in a political environment characterized by a sub-national authoritarianism that prevents the construction of dissident political agendas, and a lack of specialized knowledge about the problem of climate change by local activists. For climate science information to be assimilated, it must actively communicate in the appropriate language, seeking participatory mechanisms that generate reliable and transparent data production, and that strengthen the emergence of a common environmentalist agenda.
\end{abstract}

Keywords: climate change, environmentalism, activism, environmental policy, civil society.

\footnotetext{
- Doctorante en Ciencias Ambientales, Centro de Ciencias de Desarrollo Regional por la Universidad Autónoma de Guerrero. Orcid: 0000-0003-1676-1659.

- Doctor en Investigación en Ciencias Sociales por la Facultad Latinoamericana de Ciencias Sociales, sede México.Actualmente es catedrático del Consejo Nacional de Ciencia y Tecnología en la Universidad Autónoma de Guerrero_erick.gilmour@gmail.com—Orcid:0000-0003-1946-3921.

• Doctor en Desarrollo Regional por la Universidad Autónoma de Guerrero. Profesor-investigador de tiempo completo, base Prodep, titular "A" de la Facultad de Arquitectura y Urbanismo, Universidad Autónoma de Guerrero. Orcid: 0000-0002-2698-316X.

Fecha de recepción: 20 de agosto de 2020. Fecha de aceptación: 4 de marzo de 2021.
} 


\section{Introducción}

En todo el mundo los tomadores de decisiones se enfrentan cada vez con más frecuencia al desafío de la adaptación a un clima cambiante (Moss et al., 2013). A su vez, muchas personas pueden mencionar al menos algunas cosas que podrían hacer para mitigar el cambio climático global, pero este conocimiento en la mayoría de las ocasiones no se convierte en acción ni de mitigación ni de adaptación al fenómeno.

A pesar de los llamados científicos a la acción urgente, el cambio climático no está al inicio de la lista de prioridades de las políticas públicas ni de las preocupaciones de la sociedad. Para hacer frente a los fenómenos climáticos cada vez más violentos, se requiere adaptar prácticamente todos los sistemas construidos artificialmente de todas las regiones del mundo (IPCC, 2012).

El AR5 destaca los impactos del cambio climático a las comunidades costeras. Los pronósticos indican que el aumento del nivel del mar (SLR), la vulnerabilidad de áreas costeras bajas, islas y regiones polares (Birkmann et al., 2016; Erkens, Bucx, Dam, de Lange y Lambert, 2015). En menor medida, el impacto en el turismo costero causado por el aumento de la temperatura, la pérdida de playas debido a la erosión costera y el SLR, y la reducción del suministro de agua (Nicholls et al., 2007; Wong et al., 2014). Otros impactos costeros incluyen la migración extrema de especies, que puede afectar negativamente a las comunidades tropicales y subtropicales que dependen de éstas para su seguridad alimentaria y económica (IPCC, 2019b).

El análisis longitudinal de las encuestas de opinión pública indica que el consenso entre los expertos sobre la existencia del cambio climático antropogénico es abrumadoramente alto (IPCC, 2019a, 2019b; Tollefson, 2018); la comunidad científica mundial está al tanto de la existencia, 
posible magnitud y la prospectiva del desafío que el cambio climático puede traer a la sociedad actual.

Las percepciones públicas del riesgo que representa el cambio climático, qué tanto se encuentra la sociedad civil al tanto de las proyecciones climáticas, y cuál es el apoyo para la gestión de políticas públicas que incidan en una cartera de proyectos enfocados en la adaptación frente a estas prospectivas, varían de un país a otro (Taylor, Dessai y Bruine de Bruin, 2014) en gran medida por los diferentes contextos nacionales, étnicos y culturales que existen, pero principalmente por la marcada diferencia en la exposición a los riesgos climáticos que tiene cada uno de los países (Taylor et al., 2014).

La percepción pública del riesgo es específica de la cultura y el lugar en donde se registra (Weber y Hsee, 1999); la situación económica y la educación afectan directamente cómo la sociedad reacciona ante un evento de esta naturaleza (Yearley, 2009), y por lo tanto también se debe esperar que la percepción pública de las amenazas planteada por el cambio climático, así como el apoyo a las políticas de adaptación, varíen sustancialmente de un lado del espectro de respuesta hasta el otro entre diferentes regiones.

Para mejorar el debate público y la comunicación en general en materia de la adaptación al cambio climático, es de suma importancia comprender cómo la percepción pública sobre los riesgos generados por el cambio climático difiere de las conceptualizaciones de riesgo de los expertos involucrados en el pensamiento científico (Weber y Stern, 2011).

Las evaluaciones de riesgo de los expertos se basan en proyecciones metodológicas, muchas veces numéricas y técnicas: número de muertes, lesiones y daños a las propiedades (Slovic, Fischhoff y Lichtenstein, 1979). Por el contrario, las percepciones del público tienden a considerar mayores los riesgos cuando los peligros son relativamente 
menos familiares y evocan más emociones negativas debido a su potencial catastrófico (Slovic, 1987).

Para comunicarse de manera efectiva sobre el cambio climático y la adaptación a él, es importante comprender las creencias actuales de las personas, así como los posibles malentendidos (Bruine de Bruin y Bostrom, 2013), para de esta forma llevar hasta la audiencia la mayor cantidad de información científica ya digerida en un formato que sea tanto claro como conciso, con un vocabulario pertinente.

\section{Antecedentes}

Los Objetivos de Desarrollo Sostenible (oDs) adoptados en 2015 forman parte de la política internacional de cambio climático, en concreto, el Objetivo 13, la "Acción por el clima" promueve la adaptación de las medidas de emergencia para adaptarse al cambio climático y sus efectos (Naciones Unidas, 2015b). En cuanto a la política de México, se destaca la Ley General de Cambio Climático (LGCC) de 2012, y en 2015 el estado de Guerrero publicó la Ley número 845 de Cambio Climático del Estado de Guerrero. A pesar de ello, aún queda mucho trabajo por hacer para implementar los mecanismos adecuados, que incorporar conceptos de adaptación y vulnerabilidad a través de visiones locales, regionales y nacionales (Solorio y Miranda, 2019; Sosa-Rodríguez, 2015).

El caso concreto de Acapulco es ejemplar en este sentido. Siguiendo a autores como Giddens (2010), la gran paradoja en la formulación de políticas de cambio climático a nivel internacional es que, a pesar de que la información científica y técnica sobre el problema es vasta y con gran peso argumentativo, los tomadores de decisiones no hacen nada sustancial para mitigar o generar condiciones de adaptación al fenómeno planteado (Giddens, 2010). 
Desde el cuarto informe de evaluación del Panel Intergubernamental de Cambio Climático (IPCC, 2007) se habla de un aumento promedio de una décima de grados Celsius de temperatura por década desde los años cincuenta. Actualmente se reconoce que la temperatura del planeta ya ha aumentado un grado Celsius desde la Revolución Industrial y se espera que siga aumentando con una tasa cada vez más pronunciada, generando creciente preocupación en la comunidad científica (IPCC, 2018).

Se citan hechos contundentes: el aumento de emisiones mundiales de gases de efecto invernadero (GEI) ha sido del $70 \%$ entre 1970 y 2004 y seguirán en aumento entre un 25 y $90 \%$ a pesar de las políticas de mitigación ya llevadas a cabo por diversos países (y los Protocolos de Kioto y de París, entre otros), y de proseguir esta tasa de aumento en la emisión de GEI, los cambios en la temperatura global en el presente siglo serán probablemente mayores a los experimentados el siglo anterior (IPCC, 2013, 2014).

Cronológicamente los fenómenos hidrometereológicos de mayor relevancia en la ciudad de Acapulco de Juárez son el huracán Paulina (1997) (Lawrence, 1997), el huracán Henriette (2007) (Knabb, 2007), los eventos de mar de fondo $(2008,2015)$ (Jaimes Tenorio, Guinto Herrera y Arroyo Matus, 2017), las fuertes lluvias producto de La Niña (2010), la tormenta tropical Harvey (2011), los huracanes Ingrid y Manuel (2013) (Beven II, 2013; Pasch y Zelinsky, 2013), y las fuertes lluvias producto de El Niño (2015-2016) (L'Heureux, 2016). Según un estudio elaborado por la Secretaría de Turismo (2014), el impacto del cambio climático en Acapulco a fines de este siglo será de marejada ciclónica, aumento del nivel del mar, crecidas de ríos y refracción de olas, vientos extremos, sequías y aumento de la temperatura (Sectur, 2014).

Si bien se han hecho diversos seguimientos en la elaboración de políticas públicas para la mitigación y/o adaptación 
al cambio climático (Massey y Huitema, 2016; Wellstead y Stedman, 2015), casos en México a nivel regional (Semarnat-INECC, 2016) dan cuenta de problemáticas aún más complejas de las que menciona Giddens (2009).

A pesar de la implementación de diversos mecanismos legales para reglamentar las emisiones de GEI (Becerril, De la Parra, López Velasco y Pacha, 2019; Tejeda-Martínez, 2019):

Se desarrollan leyes ex profeso de acuerdo con coyunturas políticas específicas.

a. La preocupación por los efectos de cambio climático sólo se ha reflejado en estrategias de planeación o en programas sectoriales muy acotados, sin un interés real de los gobiernos por apoyar plenamente dichas iniciativas.

b. La relación entre las leyes estatales de cambio climático frente a la ley general en la materia es muy difusa, con muy poca armonización y con muchas lagunas legales dentro de sus esquemas operativos.

c. Las proyecciones de nivel del mar hacia finales del siglo muestran un notorio impacto en zonas de alta producción económica orientada al turismo, como es Acapulco Diamante, así como también en zonas de alta desigualdad económica. Los efectos del incremento local del mar requieren la proyección y la agregación de los componentes individuales del cambio en el nivel del mar (Le Cozannet, Garcin, Yates, Idier y Meyssignac, 2014) en cada sitio de interés. 
Ilustración 1. Incremento del nivel medio global del mar "Calamitosas"

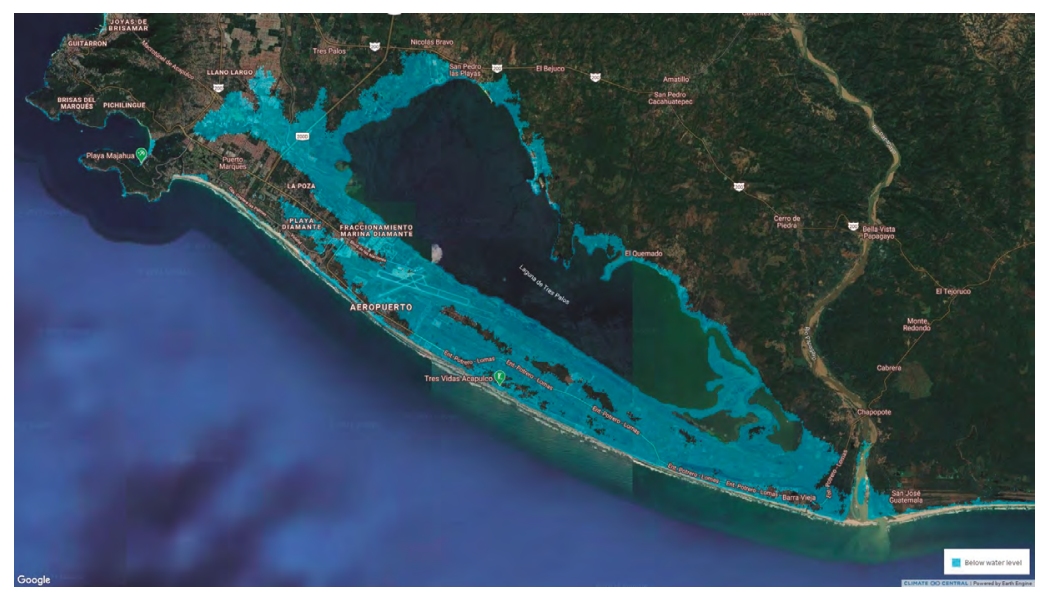

El escenario, denominado para efectos de este estudio como "calamitosas", si para el año 2100 la temperatura global del planeta aumenta solamente $+3^{\circ} \mathrm{C}$ (Kopp et al., 2014), por encima de la temperatura media del periodo entre 1850 y 1900, sin llegar a cumplir las metas del Acuerdo de París (un, 2015); por la expansión del agua del océano a medida que se calienta, por la fusión de los glaciares y la descomposición parcial de las capas de hielo de Groenlandia y la Antártida, el promedio del incremento del nivel del mar promedio en el litoral de Acapulco Diamante podría de ser en promedio de +4.7 metros, con una mínima estimada de 3 metros y una máxima esperada de 6.3 metros (B. H. Strauss, Kulp y Levermann, 2015). Según los modelos Nivel del Mar de Climate Central (Buchanan, Kopp, Oppenheimer y Tebaldi, 2016; Climate Central, 2021; Kopp et al., 2016b, 2016a; Muis, Verlaan, Winsemius, Aerts y Ward, 2016; Tebaldi, Strauss y Zervas, 2012). 


\section{Ilustración 2. Incremento del nivel medio} global del mar "in extremis"

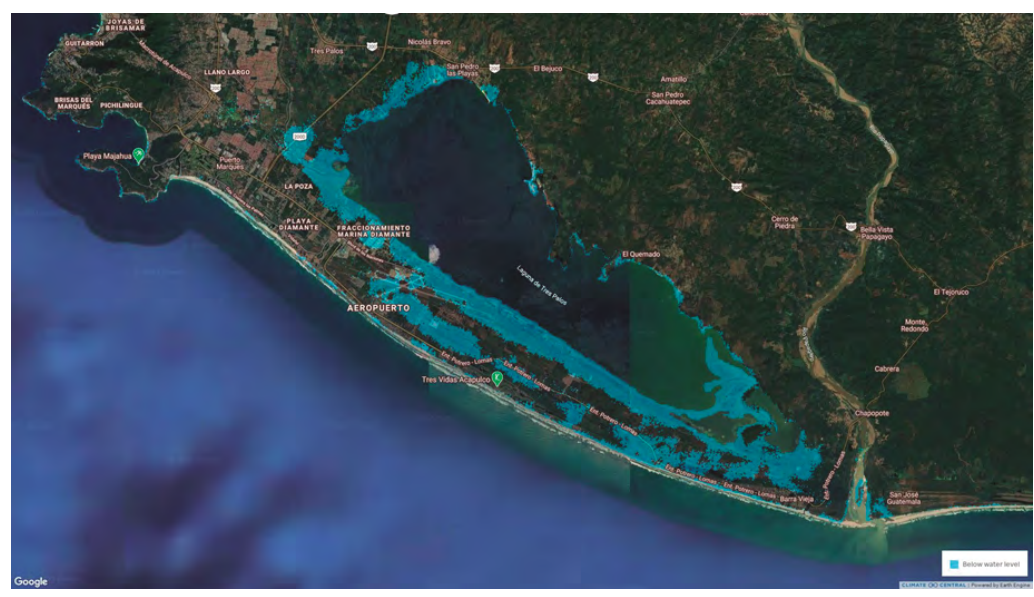

El escenario, denominado para efectos de este estudio como "in extremis", si para el año 2100 la temperatura global del planeta aumenta $+4^{\circ} \mathrm{C}$ (Kopp et al., 2017) por encima de la temperatura media del periodo entre 1850 y 1900, sin seguir ninguna de las metas del Acuerdo de París (Un, 2015) y tras experimentar cambios no lineales a gran escala en el sistema terrestre (Oppenheimer et al., 2014); por la expansión del agua del océano a medida que se calienta, por la fusión de los glaciares y la descomposición parcial de las capas de hielo de Groenlandia y la Antártida, el promedio del incremento del nivel del mar promedio en el litoral de Acapulco Diamante podría de ser en promedio de +8.9 metros, con una mínima estimada de 6.9 metros y una máxima esperada de 10.8 metros (B. H. Strauss et al., 2015). Según los modelos Nivel del Mar de Climate Central (Buchanan et al., 2016; Climate Central, 2021; Kopp et al., 2016b, 2016a; Muis et al., 2016; Tebaldi et al., 2012). 
Ante ello, toda una serie de actores de la sociedad civil acapulqueña con agenda ambientalista ${ }^{1}$ ha participado en la elaboración de propuestas y planes de ordenamiento urbano, sin que hayan podido generar incidencias claras frente a gobiernos históricamente caracterizados por una relación autoritaria y corporativista con la ciudadanía.

¿Cuáles son las herramientas cognitivas con las que cuentan los activistas ambientales en Acapulco para generar incidencia en políticas sobre cambio climático dentro de dicho municipio?

Se propone que, ante los obstáculos en la capacidad de incidencia de las organizaciones ambientalistas caracterizadas por agendas de acción divergentes, una construcción del problema orientada tanto por conocimientos de carácter científico como por percepciones de sentido común, y un evidente desencanto ante la acción de contrapeso estatal, el interés y el compromiso de los activistas frente a los escenarios de riesgo por causa del cambio climático, pueden ser factores importantes para conjuntar esfuerzos, proveer de insumos técnicos y políticos para la elaboración de políticas de mitigación frente al cambio climático en Acapulco.

Con nuestro trabajo se busca incorporar diversas reflexiones teóricas, metodológicas y empíricas que contribuyan a entender no solamente el problema de la incidencia ciudadana y la construcción social del problema del cambio climático en Acapulco, sino también para entender cómo es que los actores de la sociedad civil han desarrollado incidencia y control ciudadano hacia el Estado en un contexto

I. Para efectos prácticos, más allá de aventurarnos a hacer una definición cerrada de lo que es ambientalismo, partiremos de dos supuestos que serán importantes para entender a lo que nos referimos: I) que el ambientalismo es una agenda política desde la cual actores empresariales, ciudadanos y habitantes de un espacio determinado generan una serie de protestas y propuestas para incidir en la relación sociedad-medio ambiente, y 2) que para el presente caso, las políticas ambientales deben entenderse como una construcción social e histórica, situadas en contextos regionales específicos pero interconectadas con una necesidad global de mitigación de la devastación ecológica. 
de autoritarismo subnacional (Galán-Castro, 2018; Olvera Rivera, 2018; Valle de Bethencourt, 2019).

\section{Marco teórico}

El enfoque que se adopta en el presente estudio parte de una reflexión social de la producción del conocimiento; coincidimos con autores como Berger y Luckmann (2003), Bloor (1998) y Latour y Woolgar (1995) en cuanto a que la producción de conocimientos está mediada por un contexto social, cultural y político (Berger y Luckmann, 2003; Bloor, 1998; Latour y Woolgar, 1995). Es decir, toda empresa de generación de conocimientos — sean de sentido común, científico o incluso religioso - se realiza mediante mecanismos que otorgan a dichos saberes cualidades suficientes para adquirir facticidad objetiva (Berger y Luckmann, 2003).

Para poder elaborar un análisis de dichos mecanismos, Bloor propone una serie de principios epistemológicos que guiarían la reflexión crítica de la construcción social de conocimientos:

1. Debe ser causal, es decir, ocuparse de las condiciones que dan lugar a las creencias o a los estados de conocimiento. Naturalmente, habrá otros tipos de causas además de las sociales que contribuyan a dar lugar a una creencia.

2. Debe ser imparcial respecto a la verdad y falsedad, la racionalidad y la irracionalidad, el éxito o el fracaso. Ambos lados de estas dicotomías exigen explicación.

3. Debe ser simétrica en su estilo de explicación. Los mismos tipos de causas deben explicar, digamos, las creencias falsas y las verdaderas.

4. Debe ser reflexiva. En principio, sus patrones de explicación deberían ser aplicables a la sociología misma. Como el requisito de simetría, éste es una respuesta a la necesidad de buscar explicaciones generales. Se

\section{0}


trata de un requerimiento obvio de principio porque, de otro modo, la sociología sería una refutación viva de sus propias teorías (Bloor, 1998).

La propuesta de Bloor sobre el análisis de la producción de conocimiento nos adentra a lo que Latour y Woolgar llaman el dilema del observador: si bien el científico debe asumir una marcada diferencia entre los factores "sociales" y "científicos" de la producción de conocimientos, el analista social, como un observador externo del proceso, debe al mismo tiempo tratar su objeto de estudio con la mayor crítica posible, pero teniendo en cuenta que lo que observa es equiparable a otras formas de generar conocimiento (Latour y Woolgar, 1995).

En este sentido, la producción del conocimiento sobre el cambio climático no puede ser vista como un fenómeno aséptico, desprovisto de intereses y de puntos de vista constituidos culturalmente. Giddens menciona la existencia de un proceso paradójico, en el que la producción de conocimiento sobre el cambio climático no necesariamente ha incidido directamente en la elaboración de políticas que atiendan de raíz el problema (2010), pero nuestra apuesta teórica busca entender el carácter eminentemente situado de la producción de conocimientos y políticas sobre dicho problema (Galán-Castro, Gómez-Villerías y Torres-Rico, 2021; Giddens, 2010).

De esta manera, consideramos que no se puede desvincular la constitución de acciones políticas y conocimientos sobre el cambio climático en Acapulco sin tener como referencia una serie de factores históricos y culturales a nivel local, como el carácter vertical y autoritario de las relaciones políticas en Guerrero (Illades, 2010), y las expectativas culturales en torno al papel del Estado —desconfianza generalizada en el Poder Legislativo y los partidos políticos, preferencia por la figura de un "gran señor" con mano fuerte 
al mando del Ejecutivo, y poca confianza en la eficacia de la participación ciudadana (Téllez, 2012).

\section{Metodología}

Existen muchos métodos de análisis, pero para el propósito de este artículo se aplicó el análisis temático de corte cualitativo para realizar el análisis a través del programa Atlas.ti, versión 7.5.4 (Lewis, 2004) hacia importar la transcripción de cada entrevista en profundidad semiestructurada (documentos primarios). Atlas.ti es un programa de análisis cualitativo asistido por computadora (QDA) (Hwang, 2008).

El programa Atlas.ti se utilizó para importar la transcripción de cada entrevista y para codificar cada respuesta utilizando la estrategia de codificación abierta, axial y selectiva (A. Strauss y Corbin, 1990). La información se codificó y categorizó identificando uno o más pasajes de texto con un tema y relacionándolo con un código, que era una referencia abreviada de una idea temática (Gibbs, 2007).

Además, ofrece un proceso exhaustivo para identificar numerosas referencias cruzadas entre los temas que van surgiendo y toda la información, permitiendo vincular varios conceptos y opiniones de los participantes y compararlos con los datos que han sido guardados en diferentes situaciones en distintos momentos durante la investigación (Alhojailan y Ibrahim, 2012).

$\mathrm{Al}$ ser la entrevista en profundidad semiestructurada una herramienta de carácter cualitativo, es importante reconocer la necesidad de establecer un marco para el muestreo de los actores a entrevistar (Wilson, 2014). Al tratarse mayormente de actores provenientes de distintas disciplinas, sectores y escalas territoriales, emerge la complejidad de poder demarcar la cantidad adecuada de entrevistados

\section{2}


para poder confirmar o falsear la hipótesis propuesta (De Zengotita, 2019).

El recabado de información fue generado mediante la elaboración de 10 entrevistas en profundidad semiestructuradas a actores civiles con agenda ambientalista, con experiencia de participación dentro de consejos de planeación urbana o en consejos de cuenca de la Comisión Nacional del Agua (Conagua). Las entrevistas duraron de 30 a 65 minutos, con preguntas orientadas a definir, desde la perspectiva de los actores, qué es el cambio climático, sus causas, consecuencias, fuentes de información sobre el tema, experiencias de participación en procesos de planeación, y perspectivas sobre posibles vías de atención al problema desde la acción ciudadana y gubernamental. En algunos casos la entrevista se desarrolló en el domicilio de los informantes, y cuando esto no pudo ser posible por motivos de salud o ausencia de la ciudad de los informantes, se hizo mediante videoconferencias. El periodo de recopilación de información fue del 18 de abril al 9 de mayo de 2019 .

De todo este material se extrajo un total de 508 citas a través de la categorización de 24 códigos, de los cuales 18 fueron cerrados y seis abiertos, que emergen de los datos a través de continuas y meticulosas revisiones, y de su constante comparación, que nos permitió identificar las principales categorías, con los cuales se extrajeron cuatro supercódigos generales con el fin de establecer interconexiones entre categorías y subcategorías.

$\mathrm{El}$ análisis de las entrevistas fue un proceso altamente iterativo que implica la lectura sucesiva, codificación, revisión y recodificación de los datos en categorías o familias (Fereday y Muir-Cochrane, 2006). Este proceso usa el razonamiento inductivo, por el cual las categorías y códigos, apoyados en citas, emergen de los datos a través de un examen cuidadoso de los investigadores y de su comparación constante. Por cuestiones de protección a los informantes, 
hemos mantenido su identidad bajo reserva, por lo que hemos optado por citarlos por tipo de actor y por orden de respuesta.

\section{Resultados y discusión}

\section{Construcción discursiva del cambio climático} como escenario problemático

Se observó consistentemente a lo largo de las entrevistas, y en consonancia con otros autores (Reynolds, Bostrom, Read y Morgan, 2010), que los riesgos asociados al cambio climático a menudo se construyen en relación con otros problemas ambientales locales o regionales no formalmente atribuibles al cambio climático. Cuando se les pidió a los entrevistados que describieran qué es lo que ellos entienden por cambio climático, algunos dieron como ejemplo de los eventos de mar de fondo y/o la posible llegada de tsunamis a las costas de Acapulco:

[...] en Acapulco, yo creo que el efecto más evidente que hemos estado por sufrir son los eventos de mar de fondo [...] (P4 - actor económico). ${ }^{2}$

[...] hasta tal vez prevenir algunas inundaciones, como tsunamis, como los grandes deshielos de los polos que podrían provocar hasta inundaciones en algunas zonas de Acapulco (P6 - actor social).

El análisis de las entrevistas concuerda con la investigación bibliográfica (Fischer et al., 2012) e indica que el público en general a menudo no logra distinguir entre diferentes cuestiones ambientales, enfatizando las preocupaciones generales relacionadas con el uso de los recursos, independientemente del tema específico en cuestión:

2. Para preservar la seguridad de los participantes de las entrevistas en profundidad semiestructuradas, se han suprimido sus nombres, reemplazándolos con una clave numérica y el sector del que emanan. 
[...] hay cambios en la distribución de especies que representan que la pesca se está cambiando de lugar (P4 - actor económico).

Sí, mucho, afecta, a nosotros mismos, porque pescamos el pescado chico, no es el pescado grande como debe de ser (P5 - actor económico).

Estas observaciones son consistentes con la noción de que el pensamiento cotidiano de los actores legos se basa en atribuciones de sentido espontáneas (Stanovich y West, 2008). Cuando un individuo no tiene una sólida opinión o conocimiento sobre un tema, busca el símil más cercano y adapta su respuesta a este cuestionamiento:

[...] la contaminación de la Laguna de Tres Palos ha bajado el nivel del agua, la temperatura del agua ha sobreexcedido, ha habido mortandad de peces que de la noche a la mañana nomás salen y se paran en la playa de la laguna, ha bajado la producción pesquera (P8 - actor social).

[...] el cambio climático es un efecto calor, provocado precisamente por la contaminación humana, desde que puede ser acciones de no tirar la basura en su lugar ( $P 6$ - actor social).

El examen de las entrevistas también concuerda con la investigación bibliográfica (Wallquist, Visschers y Siegrist, 2010) que sugiere que las personas recurren a analogías con riesgos familiares cuando intentan comprender riesgos menos familiares:

Se empieza a manifestar en las zonas más desprotegidas en la periferia de la zona urbana, que es donde más se evidencia la contaminación y los efectos del cambio climático, con una vulnerabilidad espantosa (PI - actor económico). 
[...] las emisiones de los equipos para combatir el clima son los que mayor aportación hacen del medio ambiente, mayor aportación de gases, mayor aportación de contaminación (P3 - actor económico).

Para tomar las medidas de adaptación apropiadas respecto a los riesgos presentes y futuros, se debe reconocer qué es lo que está en riesgo (Fielding, 2012). Sin embargo, como la investigación lo demuestra, no todos los actores son conscientes para su área local de las amenazas que podrían constituir un riesgo:

[...] a las afueras de la ciudad en donde se presentan son más bajas vemos que ya es el efecto del cambio climático se viene manifestando en dos ríos que llegan a la zona de la ciudad (P3 - actor económico).

Pues podrían ser las zonas de planicies por elevadas, no tanto en la zona de la bahía de Acapulco, grandes cerros no podrían ser grandes efectos ni muy vulnerables ( $P 6$ - actor social).

Del mismo modo, los actores pueden ser conscientes del riesgo, pero no percibirse como vulnerables personalmente (Wolf, Adger, Lorenzoni, Abrahamson y Raine, 2010). Esto puede deberse a que las personas no se identifican, a sí mismas, como parte de un grupo que se encuentre "en riesgo":

Definitivamente los sectores más pobres, precisamente porque el asentamiento de la ciudad que se encuentra dentro de la bahía está hoy día a buen recaudo protegido hasta cierto punto debido a los últimos efectos naturales y la zona más desprotegida es la zona de crecimiento de la ciudad (P3 - actor económico).

[...] un sector que va a ser muy afectado y es pequeño que es el sector pesquero, la mayor parte de esa gente vive cerca de las instalaciones o bien embarcaciones cerca del mar $y$ han sufrido efecto cuando hemos tenido efectos o problemas o eventos de oleaje elevado (P4 - actor económico). 
También los actores ambientalistas pueden verse motivados por un cierto optimismo (Bracha y Brown, 2012), que resulta ser una creencia errónea de las posibilidades de experimentar eventos negativos en la vida y que los eventos positivos sean más altos que los de otras personas, aunque esto también puede ser positivo para la movilización y la incidencia en la medida que motiva la necesidad de cambios en el statu quo ambiental:

[...] expedición de licencias de construcción, sugiriendo en casi todos los casos las plantas de tratamiento de aguas residuales, bajando las densidades de construcción y sugiriendo un sinfín de acciones para no impactar negativamente a la ciudad (PI - actor económico).

Semarnat [Secretaría de Medio Ambiente y Recursos Naturales], Protección Civil y otras dependencias federales fueron incluidas en la planeación de la ciudad. Ahí están mencionados los estudios y qué acciones se deben de tomar para proteger en el presente y en el futuro, los efectos que puedan este cambio climático (P3 - actor económico).

[...] desde la comunidad se efectúan acciones como la limpieza de las calles, o la separación y acumulación de basura, concretamente del pet [Polyethylene terephthalate], $y$ el hacer llegar éste a algunas industrias de reciclado (P6 - actor social).

Respecto a esto último (Jiménez-Castillo y Ortega-Egea, 2015) se ha encontrado que el sesgo de optimismo está asociado con una mayor aceptación del riesgo de cambio climático entre los residentes de la costa de Acapulco de Juárez, México:

Acciones para adaptarse al cambio climático, pues algunas como y en Acapulco, hace algunos años, unos 10 años empieza a usar las fotoceldas, o celdas fotovoltaicas, y eso elimina un poquito la energía eléctrica y la contaminación (P6 - actor social). 
[...] una de las acciones es que el Gobierno del estado tiene ya en su legislación una ley sobre el cambio climático, de la cual nosotros pues estuvimos participando precisamente con algunas opiniones como sociedad civil (P2 - actor económico).

Hasta la fecha la evidencia sugiere que los actores tienden a ubicar a los actores gubernamentales como los responsables de implementar las acciones de adaptación al cambio climático:

[...] entonces sí vemos un descuido, una omisión por parte de la autoridad municipal,y un descuido total del Gobierno federal (P3 - actor económico).

[...] los organismos federales, estatales, municipales no se preocupaban, si a nivel federal no venía la visión de que [...] el ambiente era [...] había que cuidarlo, pues los de municipio y el estado menos lo hacían, bueno, hasta $[. .$.$] todavía hasta estos gobiernos anteriores lo que se asigna de$ presupuesto para los problemas ambientales, no llega ni al $1 \%$ del pib [producto interno bruto], ni siquiera (P7 - actor social).

Los testimonios muestran que la gestión del riesgo por concepto de inundación se considera una tarea para el gobierno y los legisladores, y se atribuye cierta responsabilidad a los propietarios de viviendas para proteger sus propias propiedades. En este sentido se vislumbra una visión más cercana a una relación paternalista con el Estado que la de una ciudadanía activa tendente a la contraloría social, lo cual debe entenderse en un contexto sociohistórico caracterizado por relaciones autoritarias de poder político y emergencia de poderes locales sumamente restrictivos con las disidencias (Bartra, 1996; Illades, 2010; Santiago e Illades, 2019; Valle de Bethencourt, 2019): 
[...] pero si no actuamos de forma global y las autoridades tanto municipales, estatales y federales, no se ponen las pilas, los efectos de Acapulco y de todo el litoral del estado podrín ser severas (P6 - actor social).

[...] venden los predios, se convierten en propiedad privada, y los nuevos propietarios del lugar, cuando sucede un evento hidrometeorológico severo, resulta que todo el primer piso de su casa se inunda, y como el desarrollador ya vendió (PIO - actor social).

Estábamos en cabildo, yo pedí la palabra al presidente municipal, y le dije la situación en que se encontraban las aguas marinas de Acapulco, que lo que había sucedido, que estaba muy sucio, que había una serie de vertimientos al mar que se tendrían que controlar y me dice:"Parece, parece joven [redactado], ¿quiénes son ustedes?" "Bueno, somos un grupo de amigos, doctores, profesores, directores de escuelas de buceo, empresarios, etcétera — dice-, pero no forman ninguna organización"; "No, no, no, venimos a exponerle, somos el grupo de buzos que [...] mire, organícense.Tenga personalidad jurídica porque así no" (P8 - actor social).

Vemos, en suma, que existen al menos tres problemáticas dentro de la relación sociedad civil-Estado para la incidencia en temas de cambio climático:

La relativa falta de familiaridad de las y los activistas con datos y magnitudes sobre el problema del cambio climático en lo general y en Acapulco en particular.

Una sociedad civil ambientalista que concibe el sentido de la acción estatal como una relación paternalista, en la que el Estado es responsable único de los conflictos ambientales no resueltos.

Una sociedad civil limitada por una historia sociopolítica definida por el autoritarismo y la represión de las disidencias.

No sólo se sostiene la paradoja Giddens — pocas o nulas acciones de raíz ante el cambio climático ante la abundan- 
cia del conocimiento técnico- sino que es difícil partir de la acción ciudadana y la contraloría social en un marco de oportunidades políticas cerradas para ésta y otras agendas civiles (Valle de Bethencourt, 2019).

\section{Concientización y difusión del conocimiento como alternativa}

Entre los no expertos, la creencia en la existencia del cambio climático como un fenómeno provocado por el hombre, genera diversas confusiones (Ratter, Philipp y von Storch, 2012), lo que abre un amplio abanico de oportunidades para trabajar en la difusión del problema y la generación de conciencia social:

[...] el cambio climático son las variaciones en el clima, en tiempos relativamente cortos que se deben a acciones provocadas por el hombre, que de otra manera no se darían (PI - actor económico).

Por cambio climático, yo entiendo una modificación del clima, el clima es una serie de procesos a nivel que tiene que ver con temperaturas con distribución de diferentes fenómenos y que tienen que ver con la estabilidad de la atmósfera ( $P 4$ - actor económico).

Se encontró que en la ciudad de Acapulco de Juárez existe una reciprocidad significativa entre la conciencia social sobre el cambio climático y la voluntad declarada de protegerse contra inundaciones a nivel local. A pesar de que algunos actores tienen una visión paternalista del problema, en la que el Estado es el único responsable por la acción pública para la adaptación al cambio climático, existen otros más que visualizan un papel más activo de la ciudadanía. Los ciudadanos comprometidos con la causa ambientalista deben contribuir con acciones de concientización en el espacio público, aunque no necesariamente el de la acotada esfera pública local, sino mediante las redes 
sociales, espacios que por su relativa novedad en la acotada esfera pública guerrerense cuentan con menos restricciones para la expresión de demandas y propuestas que pueden ser divergentes frente a la política ambiental oficial:

[...] yo creo que los ciudadanos deben iniciar una campaña de concientización a través de medios probados de comunicación, las redes sociales, su impacto y profundidad es evidente (PI - actor económico).

Pero sí debiéramos ya de tomar acciones, deberían ya a nivel municipal, a nivel estatal y nivel federal, distinguir los efectos que tiene el cambio climático en Acapulco (P6 - actor social).

En la literatura de estudios sobre percepciones del cambio climático, algunos autores sugieren que la familiaridad con los eventos climáticos extremos puede reducir el distanciamiento psicológico (Reser, Bradley y Ellul, 2014) y aumentar el apoyo a la política de adaptación (Fischer et al., 2012). Algunos de los actores entrevistados pudieron hacer este tipo de enlaces semánticos entre efectos generales del cambio climático y circunstancias cercanas en su cotidianidad, por lo que podemos considerarlo un elemento importante en la construcción de compromiso social y político con una agenda para la atención del cambio climático:

[...] hemos visto cómo año por año han incrementado su caudal y obviamente llegan a desbordarse en algunos eventos muy particulares (P3 - actor económico).

[...] estos esfuerzos pequeños que hacen los particulares, no se ven reflejados en la realidad de la vida cotidiana, en el momento de los eventos de alto riesgo y entonces cuando tenemos un evento de riesgo lo multiplicamos (P3 - actor económico). 
[...] hemos tenido eventos ciclónicos, muy frecuentes y con intensidades mucho más, mucho más, allá de tres, o de cuatro, en la categoría de Saffir-Simpson eran raros los eventos de cinco, y ahorita ya son bastante comunes (P4 - actor económico).

Las entrevistas con residentes locales que se vieron afectados por inundaciones provocadas por efectos hidrometereológicos severos (Bravo-Lujano, 2013; Cirilo y Lujano, 2013; OCHA-México, 2013), ${ }^{3}$ advirtieron que las autoridades locales eran responsables en tanto que la corrupción, la mala administración de los gobiernos locales y federales, y una desatención evidente hacia temas relacionados con la administración de recursos naturales, se encuentran presentes constantemente.

[...] sigo insistiendo en que los manejos en este tipo de cosas se han dado muy turbio, al menos hasta ahora con los gobiernos federales en turno, $y$ en este caso el gobierno municipal en turno ( $P 7$ - actor social).

¿Cómo permitió el municipio una construcción a la orilla de la cuenca del río? Una familia muy querida en Acapulco, se llevó toda la casa y se murió toda la familia (P8 - actor social).

Los informantes que habitan en la Zona Diamante, un área con alto riesgo hidrometeorológico, sugirieron que los participantes comúnmente se percibían a sí mismos como "impotentes" respecto a la gestión del riesgo de inundación. Existe una percepción compartida de desprotección estatal frente a eventos catastróficos. La inacción, la lenta respuesta gubernamental, así como la ilegitimidad ( $i . . .0$

3. Tal vez el más reciente hecho de desastre natural de alto impacto para la Zona Diamante es el que se dio en el mes de septiembre de 2013, cuando el paso de las tormentas tropicales Ingrid y Manuel hizo crecer el caudal de Río de la Sabana, de tal forma que inundó severamente dicho sector urbano junto con otras colonias cercanas. Las cifras oficiales mencionan que hubo 24 muertes en 17 colonias de Acapulco.

\section{2}


ilegalidad?) con la que se otorgan permisos de construcción de nuevos desarrollos habitacionales en la zona, son vistos como una amenaza para su integridad, y como una vulnerabilidad que no propiamente es natural, sino profundamente política y social.

La población debe tener una mayor información por parte de las autoridades correspondientes, nosotros como comunidad de asociación civil tenemos muy limitado nuestro poder (P2 - actor económico).

[...] toda la zona nueva de desarrollo de Diamante y toda la zona que se va a empezar a desarrollar por acá del lado de Pie de la Cuesta, pues es la costa abierta, no tiene protección de ninguna, de ninguna, bahía y ellos van a estar más, más, expuestos a, para más vulnerables a estos, a estos, efectos de oleaje (P4 - actor económico).

Si bien la narrativa individualista de la acción ciudadana, que puede resumirse en la noción de que las acciones individuales de los ciudadanos pueden generar incidencias estructurales ante un problema dado, puede verse como un elemento despolitizador de la acción ciudadana (Oraisón, 2009), consideramos que la voluntad de actores ciudadanos de partir de acciones personales para la generación de cambios puede ser una oportunidad para vincular actores cuyas agendas ambientalistas se encuentran dispersas. En este sentido, descubrimos que la percepción de que "puedo ayudar personalmente a reducir el cambio climático cambiando mi comportamiento", es un predictor significativo de la voluntad de tomar medidas de ahorro de energía:

[...] yo creo que el primer paso es cambiar nuestros conceptos arquitectónicos y aplicar arquitectura bioclimática, que responda a un menor consumo de energías, de gas y que podamos sumar en nuestros proyectos todo tipo de energías limpias (P3 - actor económico). 
[...] pero sí se toman algunas sugerencias, como la de utilizar las fotoceldas, la de utilizar menor cantidad de agua en los mingitorios, $O$ en los wc [inodoro], o en los lavamanos (P6 - actor social).

La aceptación de acciones tendentes a la adaptación para hacer frente a los efectos del cambio climático depende de la medida en que se perciba que los nuevos desarrollos se ajustan a una construcción identitaria de Acapulco como un enclave económico orientado al turismo (América Libertad Rodríguez-Herrera, Olivier-Salimé, López-Velasco y Barragán-Mendoza, 2017). Este punto es importante: la red civil ambientalista en Acapulco es muy diversa, y por su constitución histórica y relativa distancia ideológica de sus actores frente a otros movimientos sociales contrarios a la implementación de megaproyectos de desarrollo, vincula la catástrofe socioambiental con el fin del desarrollo turístico en la ciudad:

Acapulco vive del turismo, el principal sector afectado sería definitivamente el turístico ( $P 6$ - actor social).

No existe conciencia, la ciudad de Acapulco está totalmente desprotegida, ni gobierno, ni sociedad hace nada. No se hace nada ni siquiera de manera individual. El colapso yo creo que está próximo (PI - actor económico).

Es necesario poner énfasis en la importancia del miedo en la construcción social del problema del cambio climático. Si bien diversos autores evidencian el carácter desmovilizador del miedo en tanto que surge como respuesta ante una amenaza difusa y no controlable (Agudelo-López, 2016; Auyero y Swistun, 2009), puede ser también un elemento identificado por los activistas como un reto a vencer mediante acciones de información y generación de datos confiables. 
En este sentido, cuando los escenarios de cambio climático que provocan miedo se presentan sin pasos claros para la mitigación y la reducción del riesgo, se puede provocar fatalismo, impotencia y negación, pero esto puede combatirse generando mecanismos que identifiquen el problema del cambio climático más allá de las prenociones sobre el mismo:

Definitivamente mientras no tengamos cultura de lo que está sucediendo con el planeta, vamos a seguir teniendo presión para ocupar el territorio en su totalidad y además para entre comillas cubrirlo pavimentado y evitar con esto, o provocar más bien con esto, que el proceso natural del planeta no se lleve a cabo (P3 - actor económico).

Mira, yo creo que va a llegar un momento en que se van a tener que, esta misma pregunta es como para cuando ya la gente empieza a llegar a la parte defensiva de estas medidas de adaptación, ahorita estamos en una parte donde estamos entonces como de toma de conciencia, pero como que todavía no pasa, como que todavía hay mucha gente que no cree, que realmente esté ocurriendo que vaya a ocurrir o que les vaya a tocar a ellos, incluso hasta pensarlo desde el punto de vista de la siguiente generación o sea sus hijos, no creen que vaya a ocurrir porque va a haber necesidad, de esta parte de la respuesta esperada va a ocurrir cuando ya estemos prácticamente a la defensiva ( $\mathrm{P} 4$ - actor económico).

\section{Conclusiones}

Los modelos mentales que se basan en hechos a menudo incompletos, experiencias pasadas e incluso percepciones intuitivas, ayudan a moldear las acciones y el comportamiento, influyen en lo que las personas prestan atención en situaciones complicadas y definen cómo las personas se acercan y resuelven problemas. Sin darse cuenta pueden llevar a la gente a creer que los efectos del cambio climático podrían disiparse tan rápido como los efectos de otras 
formas de contaminación, reduciendo así la necesidad percibida de medidas de adaptación a más largo plazo.

Además de reducir potencialmente la preocupación por el cambio climático y la disposición a participar en actividades de mitigación, el sesgo de optimismo y la distancia psicológica también pueden afectar la disposición a participar en la adaptación proactiva a los futuros impactos del cambio climático. Por lo tanto, es posible que donde los impactos proyectados del cambio climático sean más familiares, más inmediatos y sobresalientes, las creencias sobre el cambio climático tengan un bajo impacto en la disposición a adoptar medidas de protección, proactivamente apoyar las políticas de adaptación.

Las personas generalmente tienen algunos conocimientos y creencias relevantes que les ayudan a interpretar nueva información para llegar a conclusiones. Cuando escuchan sobre el riesgo, las personas a menudo se refieren a fenómenos relacionados conocidos y asociaciones de su pasado para decidir si encuentran el riesgo amenazador o manejable. Se ha argumentado que factores como la cultura, la identidad, el apego al lugar, los valores y las actitudes de riesgo regionales determinarán tanto la necesidad percibida de adaptarse como la aceptabilidad de medidas adaptativas particulares. Algunos autores señalan la importancia de examinar cómo las percepciones de los no expertos sobre el cambio climático están relacionadas con su relación con el paisaje, y la necesidad de considerar el apego al lugar en relación con la adaptación al cambio climático.

Lo que hemos hallado es que, tanto los actores ciudadanos que hemos entrevistado, como los informes emitidos desde organismos especializados como el IPCC, coinciden en la urgencia de efectuar acciones políticas y sociales para la adaptación y mitigación de los efectos del cambio climático, especialmente en un espacio costero como lo es la ciudad de Acapulco. Tenemos una ciudadanía en alerta 
por el riesgo climático, y que a pesar de la historia de las relaciones políticas dentro de Guerrero, caracterizada por la acción autoritaria de los grupos políticos frente a las demandas ciudadanas, así como de limitadas experiencias de procesos de democratización que han sido limitadas por la precariedad organizativa de la sociedad civil y la constante amenaza de violencia política (Bartra, 1996; Illades, 2010), consideran que las acciones ciudadanas tendentes a la concientización y sensibilización sobre las consecuencias del calentamiento global pueden ser una alternativa viable para incidir en políticas de planeación urbana adecuadas a la problemática del incremento del nivel del mar.

Con un problema tan complejo como el cambio climático, los ciudadanos(as) necesitan saber que hay soluciones para enfrentarlo, y que pueden ser parte de esas soluciones. La investigación proporciona evidencia para sostener que es posible la acción ciudadana para generar contrapesos institucionales al Estado, en aras de lograr no solamente incidencia directa en la acción gubernamental en un entorno caracterizado por un histórico autoritarismo de las relaciones sociedad-Estado, sino también para la consolidación de una agenda común ambientalista que pueda fortalecer la democracia a nivel local.

Esperamos que, junto con los últimos avances en ciencias climáticas, ingeniería, economía y política ambiental, se pueda comunicar el cambio climático de una manera que resuene con su audiencia, creando conciencia social que sirva como germen de un ejercicio social más avanzado para la creación y consolidación de un observatorio ciudadano ambiental en Acapulco, Guerrero.

Tabla de ilustraciones

Ilustración I Incremento del nivel medio global del mar "Calamitosas". 3 llustración 2 Incremento del nivel medio global del mar "in extremis". 
Bibliografía

Agudelo-López,A. (2016). Fobopolítica:Rúbricas de una gubernamentalidad contemporánea. Universidad de Manizales/ CINDE.

Alhojailan, M. I., e Ibrahim, M. (20I2). Thematic Analysis: A Critical Review of Its Process and Evaluation. WEl International European Academic Conference Proceedings, I (20I I): 8-2I. http://www.westeastinstitute.com/wp-content/ uploads/2012/I0/ZG I 2-19|-Mohammed-Ibrahim-Alhojailan-Full-Paper.pdf

Auyero, J., y Swistun, D. A. (2009). Flammable: Environmental suffering in an Argentine shantytown. Oxford University Press.

Bartra, A. (1996). Guerrero bronco. Campesinos, ciudadanos y guerrilleros en la Costa Grande. Ciudad de México: Ediciones Era, Col. Problemas de México.

Becerril, H., De la Parra,A. M., López Velasco, R., y Pacha, M. J. (2019). Coyuca Resiliente al Clima. Iniciativa Ciudades Resilientes al Clima. México: Coyuca de Benítez.

Berger, P., y Luckmann, T. (2003). La construcción social de la realidad. Buenos Aires, Argentina:Amorrortu Editores.

Beven II, J. L. (20I3). Hurricane Ingrid. National Hurricane Center.Tropical Cyclone Report. (Vol.ALI02013). https:// www.nhc.noaa.gov/data/tcr/ALI02013_Ingrid.pdf

Birkmann, J., Agboola, J. I., Welle, T., Ahove, M., Odunuga, S., von Streit,J.,y Pelling, M. (20I6).Vulnerability, Resilience and Transformation of Urban Areas in the Coastal Megacity Lagos: Findings of Local Assessments and a Household Survey in Highly Exposed Areas. Journal of Extreme Events, 03(03). 16500 19. https://doi.org/ 10.1 142/ S2345737616500196

Bloor, D. (1998). Conocimiento e imaginario social. Gedisa. https://books.google.com.mx/books/about/Conocimiento_e_imaginario_social.html?id=u6xtowaacaaj\&redir_esc $=y$ 
Bracha,A.,y Brown, D.J. (20I2).Affective decision making:A Bibliografía theory of optimism bias. Games and Economic Behavior, 75(I): 67-80. https://doi.org/I0.1016/j.geb.20 I I.I I.004

Bravo-Lujano, C. (20I3). Reseña del huracán "Ingrid” del Océano Atlántico. México, DF.

Bruine de Bruin, W., y Bostrom, A. (20I3). Assessing what to address in science communication. Proceedings of the National Academy of Sciences, I I0(Supplement_3): |4062-I4068. https://doi.org//0.1073/pnas. I 2 I 2729 I I0

Buchanan, M. K., Kopp, R. E., Oppenheimer, M., y Tebaldi, C. (2016).Allowances for evolving coastal flood risk under uncertain local sea-level rise. Climatic Change, 137(3-4): 347-362. https://doi.org/ | 0.1007/s I0584-016-1664-7

Cirilo, M. G.,y Lujano, B. (20 I 3). Reseña del huracán “Manuel” del Océano Pacífico (Pp. I-9).

Climate Central. (202I). Land projected to be below tideline in 2100. Recuperado de Coastal Risk Screening Tool: https://coastal.climatecentral.org/ map/l 3/-99.7/54//6.7525/?theme=sea_level_ rise\&map_type=year\&basemap=hybrid\&contiguous=true\&elevation_model=best_available\&forecast_year $=2100 \&$ pathway $=r c p 26 \&$ percentile $=p 50 \&$ refresh=true\&return_level=return_level_0\&sIr_model

De Zengotita,T.(2019). Critical Theory. Political Philosophy and Public Purpose (pp. I27-I36). https://doi.org/ I0.1007/9783-319-90689-8_7

Erkens, G., Bucx,T., Dam, R., de Lange, G., y Lambert,J.(20I5). Sinking coastal cities. Proceedings of the International Association of Hydrological Sciences, núm. 372, pp. I89- 198. https://doi.org//0.5194/piahs-372-189-2015

Fereday,J.,y Muir-Cochrane, E. (2006). Demonstrating Rigor Using Thematic Analysis: A Hybrid Approach of Inductive and Deductive Coding and Theme Development. International Journal of Qualitative Methods, 5(I): 80-92. https://doi.org/I0.1 I77//60940690600500I07 
Bibliografía

Fielding, J. L. (2012). Inequalities in exposure and awareness of flood risk in England andWales. Disasters, 36(3): 477494. https://doi.org/I 0. I I I I/j. I467-77I 7.20I I.0I270.x

Fischer,A., Peters,V., Neebe, M.,Vávra,J., Kriel,A., Lapka, M.,y Megyesi, B. (20I2). Climate Change? No,Wise Resource Use is the Issue: Social Representations of Energy, Climate Change and the Future. Environmental Policy and Governance, 22(3): 16I-I76. https://doi.org/10.1002/ eet. 1585

Galán-Castro, E.A. (20/8). La coproducción de seguridad en Veracruz: Un enfoque morfogenético. Interregnos Subnacionales. La implementación de la Reforma al Sistema Penal en México (pp. 339-365).

Galán-Castro, E.A., Gómez-Villerías, R. S., y Torres-Rico, R. (202I). Campos de acción estratégica, ambientalismo y participación en Acapulco, Guerrero. Revista Mexicana de Estudios de los Movimientos Sociales, 5(I): 23.

Gibbs, G. (2007). Analyzing Qualitative Data. Qualitative Research Kit. https://doi.org/I0.4I35/978I849208574 Giddens, A. (2009). La paradoja de Giddens. The Politics of Climate Change, Cambridge, núm. 264. https://dialnet. unirioja.es/servlet/articulo?codigo $=3184234$

-.(2010).La política del cambio climático (I a edición).https:// www.alianzaeditorial.es/libro.php?id=2336020\&id col $=100508 \&$ id_subcol $=100520$

Hwang, S. (2008). Utilizing qualitative data analysis software: A review of Atlas.ti. Social Science Computer Review, 26(4): 5 | 9-527. https://doi.org/ I0. I 177/08944393073 I 2485

Illades, C. (2010). Breve historia de Guerrero (2 ${ }^{\mathrm{a}}$ edición). Fondo de Cultura Económica/Fideicomiso Historia de las Américas/El Colegio de México.

IPCC. (2007). Climate Change 2007: The Physical Science Basis. En S. Solomon, D. Qin, M. Manning, Z. Chen, M. Marquis, K. B.Averyt, ... y H. L. Miller (eds.), Contribution of Working Group I to the Fourth Assessment Report of 
the Intergovernmental Panel on Climate Change. (Edición 2007). https://doi.org/I0.1017/CBO978 I I074I5324.004 . (20I2). Managing the Risks of Extreme Events and Disasters to Advance Climate ChangeAdaptation. En C. B. Field, V. Barros, T. F. Stocker, D. Qin, D. J. Dokken, K. L. Ebi, ... y P. M. Midgley (eds.), A Special Report ofWorking Groups I and II of the Intergovernmental Panel on Climate Change. (Edición 2012). https://doi.org/I0.I596/978-082। 3-8845-7

_- (20I3). Climate Change 20I3: The Physical Science Basis. En T. F. Stocker, D. Qin, G.-K. Plattner, M.Tignor, S. K. Allen, J. Boschung, ... y P. M. Midgley (eds.), Contribution of Working Group I to the Fifth Assessment Report of the Intergovernmental Panel on Climate Change. (Edición 20 I3). https://doi.org/ I0. I0 I 7/cbo978 I I074 I5324.005 _. (20I4). Climate Change 20I4: Mitigation of Climate Change. En O. Edenhofer, R. Pichs-Madruga, Y. Sokona, E. Farahani, S. Kadner, K. Seyboth, ... y J. C. Minx (eds.), Contribution of Working Group III to the Fifth Assessment Report of the Intergovernmental Panel on Climate Change. (Edición 2014). Cambridge, Reino Unido: Cambridge University Press.

- (2018). Global Warming of I.5 $5^{\circ}$ C. En V. Masson-Delmotte, P. Zhai, H.-O. Pörtner, D. Roberts, J. Skea, P. R. Shukla, ... y T.Waterfield (eds.), An IPCC Special Report on the impacts of global warming of $1.5^{\circ} \mathrm{C}$ above pre-industrial levels and related global greenhouse gas emission pathways, in the context of strengthening the global response to the threat of climate change, sustainable development. (Vol.85). . (2019a). Climate Change and Land. En P. R. Shukla, J. Skea, E. C. Buendia, V. Masson-Delmotte, H.-O. Pörtner, D. C. Roberts, ... y J. Malley (eds.), IPCC special report on climate change, desertification, land degradation, sustainable land management, food security, and greenhouse gas fluxes in terrestrial ecosystems (p. 40). https://www.ipcc.ch/srccl/ 
Bibliografía
- (2019b). Ocean and Cryosphere in a Changing Climate. En H.-O. Pörtner, D. C. Roberts,V. Masson-Delmotte, P. Zhai, M.Tignor, E. Poloczanska, ... y N. M. Weyer (eds.), IPCC Special Report on the Ocean and Cryosphere in a Changing Climate (p. 34). https://www.ipcc.ch/srocc/ Jaimes Tenorio, C. I. ., Guinto Herrera, E. R., y Arroyo Matus, R. (2017). Mar de fondo: Propuesta para mitigar inundaciones en la calle Juan Serrano de la ciudad de Acapulco, Guerrero, México. Investigación y Ciencia, 25(72): 75-8I. Jiménez-Castillo, D., y Ortega-Egea, J. M. (2015). Too positive to change? Examining optimism bias as a barrier to media effects on environmental activism. Journal of Environmental Psychology, núm. 43, pp. 216-225. https:// doi.org/10.1016/j.jenvp.20I5.07.004

Knabb, R. D. (2007). Hurricane Henriette. Tropical Cyclone Report. https://www.nhc.noaa.gov/data/tcr/ep I I 2007 henriette.pdf

Kopp, R. E., DeConto, R. M., Bader, D.A., Hay, C. C., Horton, R. M., Kulp, S., ... y Strauss, B. H. (20I7). Evolving Understanding of Antarctic Ice Sheet Physics and Ambiguity in Probabilistic Sea Level Projections. Earth's Future, 5(I2): I2 I7-I233. https://doi.org/ I0.1002/20 I7EF000663

Kopp, R. E., Horton, R. M., Little, C. M., Mitrovica,J.X., Oppenheimer, M., Rasmussen, D. J., ... y Tebaldi, C. (20I4). Probabilistic 21 st and 22nd century sea-level projections at a global network of tide-gauge sites. Earth's Future, 2(8): 383-406. https://doi.org/ I0.1002/20 I 4EF000239

Kopp, R. E., Kemp, A. C., Bittermann, K., Horton, B. P., Donnelly, J. P., Gehrels, W. R., ... y Rahmstorf, S. (20I6a). Correction for Kopp et al., Temperature-driven global sea-level variability in the Common Era. Proceedings of the National Academy of Sciences, I I 3(38): E5694-E5696. https://doi.org// 0.1073/pnas.1613396/ I3

—. (2016b). Temperature-driven global sea-level variability in the Common Era. Proceedings of the National 
Academy of Sciences, I I 3(I I): E I 434-E I 44 I. https://doi. org/10.1073/pnas. I5I7056II3

L'Heureux, M. (20I6).The 20I5-I6 El Niño. 4 I st NOAA Annual Climate Diagnostics and Prediction Workshop. Orono, ME.

Latour, B., y Woolgar, S. (1995). Laboratory life:The construction of scientific facts. Laboratory Life:The Construction of Scientific Facts. Princeton University Press.

Lawrence, M. B. (1997). Hurricane Pauline. National Hurricane Center.Tropical Cyclone Report. https://www.nhc.noaa.gov/ data/tcr/EPI81997_Pauline.pdf

Le Cozannet, G., Garcin, M.,Yates, M., Idier, D., y Meyssignac, B. (20I4). Approaches to evaluate the recent impacts of sea-level rise on shoreline changes. Earth-Science Reviews, núm. 138, pp. 47-60. https://doi.org/10.1016/j. earscirev.2014.08.005

Lewis, R. B. (2004). NVivo 2.0 and Atlas.ti 5.0:A Comparative Review of Two Popular Qualitative Data-Analysis Programs. Field Methods, I6(4): 439-464. https://doi. org/I0.1 I77/I525822X04269174

Massey, E., y Huitema, D. (20I6). The emergence of climate change adaptation as a new field of public policy in Europe. Regional Environmental Change, 16(2): 553-564. https://doi.org/ 10.1007/s I0I I3-0I5-077I-8

Moss, R. H., Meehl, G. A., Lemos, M. C., Smith, J. B., Arnold, J. R., Arnott, J. C., ... y Wilbanks, T. J. (20I3). Hell and High Water: Practice-Relevant Adaptation Science. Science, 342(6159): 696-698. https://doi.org/I0.II26/ science. 1239569

Muis, S., Verlaan, M., Winsemius, H. C., Aerts, J. C. J. H., y Ward, P.J.(20I6).A global reanalysis of storm surges and extreme sea levels. Nature Communications, 7(I): I 1969. https://doi.org/ I0.1038/ncomms I I 969

Nicholls, R. J., Poh, W., Burkett, V. R., Codignotto, J., Hay, J., McLean, R., ... y Woodroffe, C. D. (2007). Coastal systems and low-lying areas. En P.J.van der L., C.E.H.M.L. 

2007: Impacts, Adaptation andVulnerability. Contribution of Working Group II to the Fourth Assessment Report of the Intergovernmental Panel on Climate Change (pp. 3 15-356). http://ro.uow.edu.au/scipapers/ I64/

Ocha-México. (20/3). México: Info Note Remnants of “Ingrid" and "Manuel”. National Disaster Response Advisor, 2, 18 sept., Pp. I-2.

Olvera Rivera, A. (2018). Gestación y crisis del régimen político autoritario en Veracruz. En A. Olvera Rivera (ed.), Veracruz en su laberinto:Autoritarismo, crisis de régimen y violencia en el sexenio de Javier Duarte (Primera). Universidad Veracruzana.

Oppenheimer, M., Campos, M.,Warren, R., Birkmann,J., Luber, G., O’Neill, B.,y Takahashi, K. (20I4). Emergent risks and key vulnerabilities. En C. B. Field,V.R. Barros, D.J.Dokken, K. J. Mach, M. D. Mastrandrea, T. E. Bilir, ... y L. L.White (eds.), Climate Change 20 I4: Impacts, Adaptation, andVulnerability. Part A: Global and Sectoral Aspects. Contribution of Working Group II to the Fifth Assessment Report of the Intergovernmental Panel on Climate Change (Climate Ch). Cambridge, Reino Unido: Cambridge University Press. Oraisón, M.M.(2009). La inclusión política. La tensión individuación: Participación en la construcción de ciudadanía. XXVII Congreso de la Asociación Latinoamericana de Sociología, pp. 0-I I. Buenos Aires, Argentina: VIII Jornadas de Sociología de la Universidad de Buenos Aires.

Pasch, R. J., y Zelinsky, D. A. (20I3). Hurricane Manuel. National Hurricane Center. Tropical Cyclone Report. (Vol. EPI320I3). https://www.nhc.noaa.gov/data/tcr/ EPI320I3_Manuel.pdf

Ratter B., M. W., Philipp, K. H. I., y von Storch, H. (20I2). Between hype and decline: Recent trends in public perception of climate change. Environmental Science 
\& Policy, núm. 18, pp. 3-8. https://doi.org/10.1016/j. Bibliografía envsci.20II.12.007

Reser, J. P., Bradley, G. L., y Ellul, M. C. (20I4). Encountering climate change:"Seeing" is more than "believing." Wiley Interdisciplinary Reviews: Climate Change, 5(4): 52I-537. https://doi.org// 0.1002/wcc.286

Reynolds, T.W., Bostrom,A., Read, D., y Morgan, M. G. (2010). Now What do People Know About Global Climate Change? Survey Studies of Educated Laypeople. Risk Analysis, 30(10): I520-I538. https://doi.org/I0.1 I I I/ j.1539-6924.2010.01448.x

Rodríguez-Herrera, América Libertad, Olivier-Salimé, B., López-Velasco, R., y Barragán-Mendoza, C. (2017). Construcción de saberes en un entorno vulnerable de la periferia de Acapulco. Percepción de riesgo frente a los problemas ambientales en Llano Largo. En America Libertad Rodríguez-Herrera, B. Olivier-Salimé, y R. López-Velasco (eds.), El desarrollo sustentable: Desafios y oportunidades (I a edición, PP. 177-192). México, DF: Plaza y Valdés.

Santiago, T., e Illades, C. (2019). La guerra irregular en Guerrero, 2007-2017. Relaciones Estudios de Historia y Sociedad, 40(I57): 2007-20 I 7. https://doi.org// 0.2490I/ rehs.v40il 57.363

Sectur. (20|4).Vulnerabilidad del destino turístico Acapulco.

Estudio de la vulnerabilidad y programa de adaptación ante la variabilidad climática y el cambio climático en 10 destinos turísticos estratégicos, así como propuesta de un sistema de alerta temprana a eventos hidrometeorológicos extremos (p. 3I).http://www.sectur.gob.mx/wp-content/ uploads/2014/09/seccion-ii.-acapulco.pdf

Semarnat-INECC. (20I6). Mexico's Climate Change Mid-Century Strategy. Ciudad de México.

Slovic, P. (1987). Perception of risk. Science, 236(4799): 280285. https://doi.org// 0.I I 26/science.3563507 
Bibliografía

Slovic, P., Fischhoff, B., y Lichtenstein, S. (1979). Rating the Risks. Environment: Science and Policy for Sustainable Development, 2 I (3): I4-39. https://doi.org/I0.1080/00 I 39|57.1979.993309।

Stanovich, K. E., yWest, R. F. (2008). On the relative independence of thinking biases and cognitive ability. Journal of Personality and Social Psychology, 94(4): 672-695. https:// doi.org// 0. I037/0022-35/4.94.4.672

Strauss, A., y Corbin, J. M. (1990). Basics of qualitative research: Grounded theory procedures and techniques. PsycNET. En A. Strauss y J. M. Corbin (eds.), Qualitative Research. (4a edición). https://psycnet.apa.org/ record// 990-98829-000

Strauss, B. H., Kulp, S., y Levermann, A. (20I5). Carbon choices determine us cities committed to futures below sea level. Proceedings of the National Academy of Sciences, I / 2(44): I 3508- | 35 | 3. https://doi.org//0.1073/ pnas. 1511186112

Taylor, A. L., Dessai, S., y Bruine de Bruin, W. (20I4). Public perception of climate risk and adaptation in the UK: $\mathrm{A}$ review of the literature. Climate Risk Management, 4(5): I- I6. https://doi.org//0.1016/j.crm.2014.09.00 I

Tebaldi, C., Strauss, B. H., y Zervas, C. E. (20I2). Modelling sea level rise impacts on storm surges along us coasts. Environmental Research Letters, 7(I): 014032 . https://doi. org/10.1088/I748-9326/7///014032

Tejeda-Martínez,A. (2019).Veracruz ante el cambio climático: Acciones mínimas 2019-2024. En I. R. Méndez-Pérez, C.A. Ochoa Martínez, y C. M.Welsh Rodríguez (eds.), Programa de Estudios de Cambio Climático de la Universidad Veracruzana (PECCUV). Universidad Veracruzana: https://www.uv.mx/noticias/files/2019/03/veracruz-ante-el-cc-propuestas-mínimas-2019-2024.pdf

Téllez, S. E. (2012). Participación electoral, instituciones y cultura política en Acapulco, Gro. Journal of Chemical 
Information and Modeling, núm. 26. http://www.ieepco. org.mx/biblioteca_digital/somee 20I I/Área temática I/Téllez. Participación electoral...pdf

Tollefson, J. (2018). Clock ticking on climate action. Nature, 562(7726): I 72 y I73. https://doi.org/ I0.1038/d4 I586018-06876-2

UN. (2015). Paris Agreement/Framework Convention on Climate Change. 2 Ist Conference of the Parties (p. 3). https://doi.org/fccc/cp/2015/.9

Valle de Bethencourt, P. (2019). La violencia en Acapulco desde la sociedad civil: Mecanismos de cohesión y contraloría social. Intersticios sociales.

Wallquist, L.,Visschers V., H. M., y Siegrist, M. (20I0). Impact of Knowledge and Misconceptions on Benefit and Risk Perception of ccs. Environmental Science \& Technology, 44(17): 6557-6562. https://doi.org/ I0.102I/es I0054I2

Weber, E. U., y Hsee, C. K. (1999). Models and mosaics: Investigating cross-cultural differences in risk perception and risk preference. Psychonomic Bulletin \& Review, 6(4): 6I I-6I7. https://doi.org/ 10.3758/BF032 I 2969

Weber, E. U., y Stern, P. C. (20I I). Public understanding of climate change in the United States. American Psychologist, 66(4): 315-328. https://doi.org// 0.1037/a0023253

Wellstead,A.,y Stedman, R.(2015). Mainstreaming and Beyond: Policy Capacity and Climate Change Decision-Making. Michigan Journal of Sustainability, 3(20181221): 47-63. https://doi.org/ / 0.3998/mjs. I 23337/ 2.0003.003

Wilson, C. (20I4). General Interviewing Issues. Interview Techniques for ux Practitioners (pp. I I 3- I I 7). https://doi. org/I0.1016/B978-0-12-410393-1.00006-5

Wolf,J.,Adger,W. N., Lorenzoni, I.,Abrahamson,V.,y Raine, R. (2010). Social capital, individual responses to heat waves and climate change adaptation: An empirical study of two UK cities. Global Environmental Change, 20(I): 44-52. https://doi.org//0.1016/j.gloenvcha.2009.09.004 
Bibliografía $\quad$ Wong, P. P., Losada, I. J., Gattuso, J.-P., Hinkel, J., Khattabi, A., Mclnnes, K. L., ... y Sallenger,A. (20 I4). Coastal Systems and Low-Lying Areas. En Christopher B. Field,V.R. Barros, D.J.Dokken, K.J.Mach, y M.D. Mastrandrea (eds.), Climate Change 2014 Impacts,Adaptation, andVulnerability (Pp. 36I 410). https://doi.org/I0.1017/CBO978I I07415379.010

Yearley, S. (2009). Sociology and Climate Change after Kyoto. Current Sociology, 57(3): 389-405. https://doi. org/I0.1I77/001I392108I0I589 